
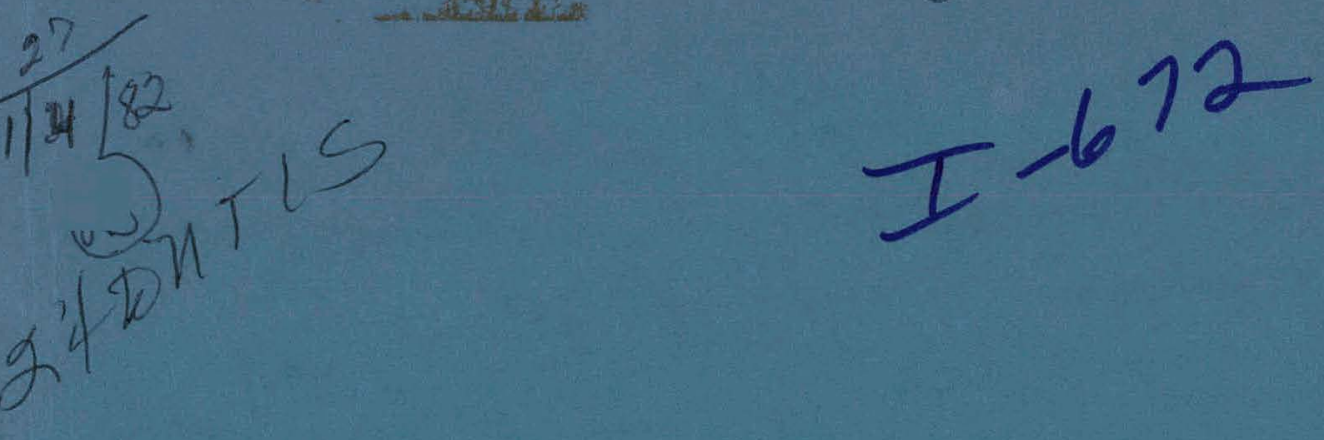

\title{
DIRECTORY OF CONTRACTORS SUPPORTED BY DIVISION OF NUCLEAR PHYSICS
}

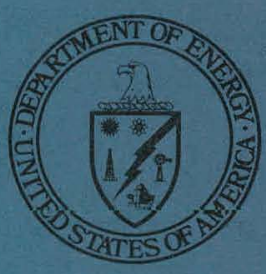

DECEMBER 1981 OFFICE OF ENERGY RESEARCH DIVISION OF NUCLEAR PHYSICS 


\section{DISCLAIMER}

This report was prepared as an account of work sponsored by an agency of the United States Government. Neither the United States Government nor any agency Thereof, nor any of their employees, makes any warranty, express or implied, or assumes any legal liability or responsibility for the accuracy, completeness, or usefulness of any information, apparatus, product, or process disclosed, or represents that its use would not infringe privately owned rights. Reference herein to any specific commercial product, process, or service by trade name, trademark, manufacturer, or otherwise does not necessarily constitute or imply its endorsement, recommendation, or favoring by the United States Government or any agency thereof. The views and opinions of authors expressed herein do not necessarily state or reflect those of the United States Government or any agency thereof. 


\section{DISCLAIMER}

Portions of this document may be illegible in electronic image products. Images are produced from the best available original document. 


\title{
DISCLAIMER
}

\begin{abstract}
"This report was prepared as an account of work sponsored by an agency of the United States Government. Neither the United States Government nor any agency thereof, nor any of their employees, makes any warranty, express or implied, or assumes any legal liability or responsibility for the accuracy, completeness, or usefulness of any information, apparatus, product, or process disclosed, or represents that its use would not infringe privately owned rights. Reference herein to any specific commercial product, process, or service by trade name, trademark, manufacturer, or otherwise, does not necessarily constitute or imply its endorsement, recommendation, or favoring by the United States Government or any agency thereof. The views and opinions of authors expressed herein do not necessarily state or reflect those of the United States Government or any agency thereof."
\end{abstract}

This report has been reproduced directly from the best available copy.

Available from the National Technical Information Service, U. S. Department of Commerce, Springfield, Virginia 22161.

Price: l'rinted Copy $\Lambda 07$

Microfiche A01

Codes are used for pricing all publications. The code is determined by the number of pages in the publication. Information pertaining to the pricing codes can be found in the current issues of the following publications, which are generally available in most libraries: Energy Research Abstracts, (ERA); Government Reports Announcements and Index (GRA and I); Scientific and Technical Abstract Reports (STAR); and publication, NTIS-PR-360 available from (NTIS) at the above address. 
DOE/ER--0114

DE82 005431

\section{DIRECTORY OF CONTRACTORS SUPPORTED BY \\ DIVISION OF NUCLEAR PHYSICS}
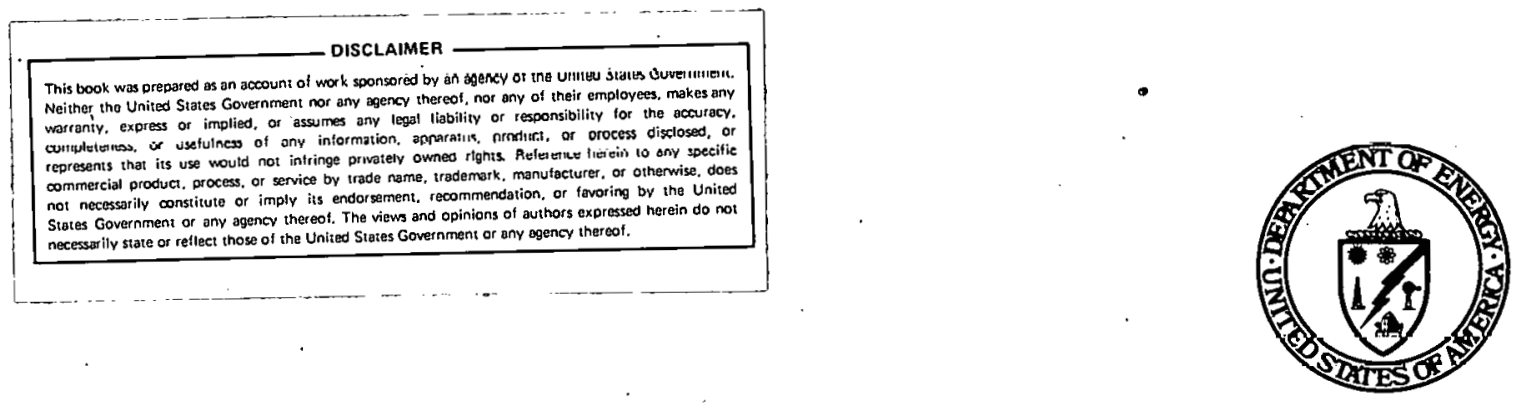

DECEMBER 1981

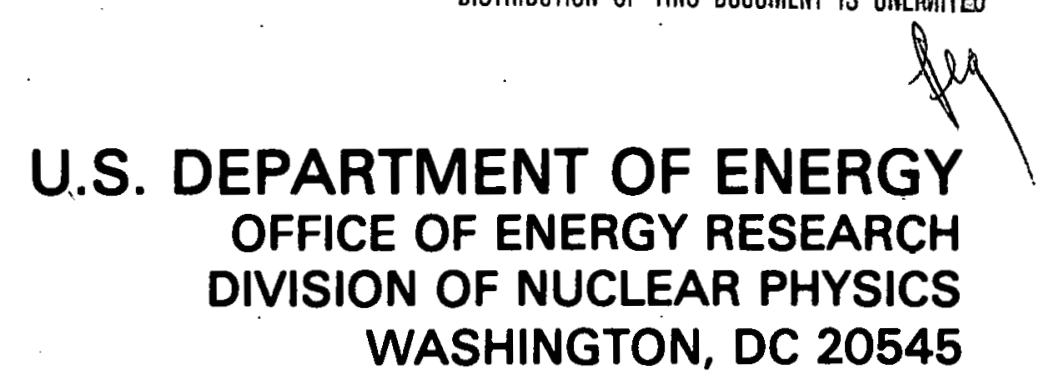


Institution: Abillene Christian University

Principal Investigator(s): M. Sadler

Contract NO.: DE-ACO5-81ER40036

Title: "Precision Measurements of Pion-Nuclear Elastic Scattering in the $\mathrm{P}^{3}$ Region at LAMPF"

Address: Department of Physics

Abilene Christian University

Abilene, TX 79699

Phone Number: 505-662-6321 (home) 505-667-6533.

Personnel (September 1981):

Faculty:

M. Sadler

Post-doctoral

Graduate Student: 
Laboratory: Ames Laboratory

Division: Nuclear Physics Theory

Personnel (September 1981):

Permanent Staff:

B. Dalton

J. $\mathrm{Hill}{ }^{*}$

J. $\operatorname{Vary}{ }^{\star}$

S. Williams

F. Wohn

A. Wolf

Post-doctorals and Visitors:
Address: Ames Laboratory Iowa State University Ames, Iowa 50011

Phone: (515)-294-2770

(FTS) $-865-2770$
A. Klar
F. Margetan
H. Yamamoto

Graduate Students:

R. Evans

M. Kiefer

D. Margetan

M. Mercier

*Person in charge 
Laboratory: Argonne National Laboratory

Division: High Energy. Physics

Personnel (September 1981):

Permanent Staff:

D. Hill

H. Spinka

A. Yokosawa

Post-doctoral and Visitors:
W. Ditzler
K. Imai
R. Stanek
K. Toshioka
R. Wagner

Address: Argonne National Laboratory 9700 South Cass Avenue Argonne, IL 60439

Phone: . (312) 972-2000

(FTS) $972-2000$ 
Laboratory: Argonne National Laboratory

Division: Chemistry

Personnel (September 1981):

Permanent Staff:

I. Ahmad

G. Bach

R. Betts

R. Chasman

P. Day

A. Friedman

J. Gindler.

L. Glendenin

S. Kaufman

E. Steinberg

J. Unik ${ }^{*}$

B. Wilkins

K. Wolf

Post-doctoral and Visitors:

B. Glagola

S. Saini
Address: Argonne National Laboratory 9700 South Cass Avenue Argonne, IL 60439

Phone: (312) $972-2000$

(FTS) $972-2000$ 
Laboratory: Argonne National Laboratory

Division: Physics

Personnel (September 1981):

Permanent Staff:
A. Bodmer
R. Holt
F. Mooring
L. Bollinger
R. Janssens
R. Pardo
F. Coester
H. Jackson
M. Peshkin
C. Davids
T. Khoo
S. Pieper
B. Day
D. Kovar
V. Krohn
D. Kurath
W. Kutschera
R. Lawson
$T$. Lee
. Henning
R. Holland
G. Garvey
D. Geesaman
K. Rehm
G. Ringo
J. Schiffer *
K. Shepard
H. Takeda
J. Yntema
B. Zeidman

Address: Argonne National Laboratory 9700 South Cass Avenue Argonne; IL 60439

Phone: (372) $972-2000$

(FTS) $972-2000$

Post-doctoral and Visitors:
D. Ashery
W. Kuhn
P. Chowdhury
H. Lipkin
H. Ernst
J. Parmentola
W. Freeman
Z. Grabowski
T. Humanic

Graduate Students:
A. Davis
B. Fillippone
S. Levenson
C. Gagliardi 
Institution: Boston University

Principal Investigator(s): G. Epstein

Contract NO.: DE-ACO2-81ER40040

Title: "Theoretical Study of Pion and Kaon Photoproduction of Nuclei"

Address: $\quad$ Department of Physics Boston University

Boston, MA 02215

Phone Number: $(617)-353-3420$

Personnel (September 1981):

Faculty:

G. Epstein

Post-doctoral:

Graduate Student:

T. Herlich 
Laboratory: Brookhaven National Laboratory

Department: Chemistry

Personnel (September 1981):

Permanent Staff:

Y. Chu

B. Cleveland

J. Cumming

R. Davis

G. Harbottle

P. Haustein

J. Hudis*

S. Katcoff

L. Remsberg

Post-doctorals and Visitors:
Address: Brookhaven National Lab. Associated Universities Inc. Upton; NY 11973

Phone: (516) 345-2123

(FTS) $666-2123$

P. Ghosh

S. Mirzadeh

P. Saha

*Person in charge 
Laboratory: Brookhaven National Laboratory

National $\mathrm{N}$ uclear Data Center

Personnel (September 1981):

Permanent Staff:

M. Bhat

T. Burrows

M. Divadeenam

C. Dunford

N. Holden

R. Kinsey

S. Mughabghab

S. Pcarlstcin*

L. Peker

S. Ramavataram
Address: Brookhaven National Lab Associated Universities Inc. Upton, NY 19973

Phone: (516) 345-2123

(FTS) 666-2123

*Person in charge 
Laboratory: Brookhaven National Laboratory

Department: Physics

Personnel (September 1981):

Permanent Staff:
A. Aerts
C. Dover
D. Alburger
R. Gill
C. Baktash
0. Hansen
A. Baltz
S. Kahana
J. Barrette
W. Kane
P. Bond
0 . Kistner
R. Casten
M. LeVine
C. Chasman
M. May
R. Chrien
D. Millener
E. derMateosian

Address: Brookhaven National Lab.Associated Universities Inc. Upton, NY 11973

Phone: (516) 345-2123

FTS $666-2123$

Post-doctorals and Visitors:
M. Collins
D. Maurizio

Graduate Students:

D. Abriola

H. Dejbaklsh

C. Ordoney

H. Al-Juwair

S. Faller

R. Rieder

M. Al-Kofahi

R. Grace

A. Smith

A. Aprahamian

R. Hackenberg

R. Wan-Yusoff

R. Boisseau

M. Marte1 
Institution: Brown University
Principal Investigator(s): S. Fallieros, F. Levin

Contract NO.: DE-ACO2-81ER0 3235

Title: "Nuclear Excitations and Reaction Mechanisms"

Address: $\quad$ Department of Physics

Brown University

Providence, RI 02912

Phone Number: (401)-863-2621

Personne 1 (September 1981):

Faculty:

S. Fallieros

F. Levin

Post-doctoral:

z. Kurouglu

Graduate Student:

R. Kozack

J. Shertzer 
Institution: University of California at Berkeley

Principal Investigator(s): K. Crowe.

Contract NO.: DE-ASO3-81ER40004

Title: "Medium Energy Meson Physics"

Address: Department of Physics University of California Berkeley; CA 94720

Phone Number: (415)-486-4000

Personnet (September 1981):

Faculty:

$\mathrm{K}$. Crowe

Post-doctoral:

R. Bossingham

A. Chacon

R. De Renzi

S. Rosenblum

E. YOO

\section{Graduate Student:}

c. Clawson

w. Zajc 
Institution: University of California, Davis

Principal Investigator(s): F. Brady

Contract NO.: DE-ACO2-81ER40037

Title: "Equipment Proposal for Bevalac Experiments"

$\begin{array}{ll}\text { Address: } & \text { Physics Department } \\ & \text { University of California } \\ & \text { Davis, CA } 95616\end{array}$

Phone Number: (916)-752-1793/1460

Personnel (September 1981):

Faculty:

F. Brady

Post-doctoral:

J. Romero

A. Sagle

Graduate Student:

G. Barasch

M. Johnson 
Institution: University of California, Los Angeles

Principal Investigator(s): R. Haddock, B. Nefkens

Contract No: DE-AT03-81ER40021

Titîe: "Medium Energy Particle Physics" (Task \#1)

"Particle Physics" (Task \#2)

Address: Department of Physics

University of California

405 Hilgard Avenue

Los Angeles, CA 90024

Phone Number: (213)-825-1993/4970

Personnel (Septeriber 1981):

Faculty:

$R$. Haddock

留

B. Nefkens

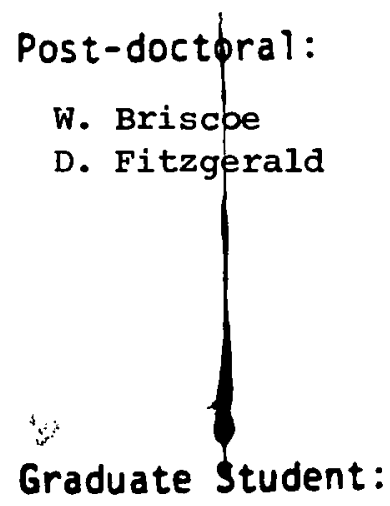

F. Borscherding

A. Eiclion

1. Mohktari

i. Schmitz

B. Silverman 
Institution: University of California, Los Angeles

Principal Investigator(s): G. Igo, c. Whitten

Contratt NO.: DE-ATO3-79ER70024

Title: "Intermediate Energy Nuclear Physics Users Group"

Address: Department of Physics

Iniversi.ty of California

Los Angeles, CA 90024

Phone Number: (213)-825-1306

Personnel (September 1981):

Faculty:

J. Carroll

G. Igo

C. Whitten

Post-doctoral:

B. Aas

E. Bleszynski

M. Bleszynski

\section{Graduate Student:}
S. Abachi
A. Azizi
K. Ganezer
A. Rahbar
J. Wagner
G. Weston 
Institution: University of California, Riverside

Principal Investigator(s): S. Fung, R. Poe

Contract NO.: DE-ASO3-81ERI0626

Title: "Central Collisions of Heavy Ion Physics"

$\begin{array}{ll}\text { Address: } & \text { Department of Physics } \\ & \text { University of California } \\ & \text { Riverside, CA } 92521\end{array}$

Phone Number: (714)-796-1277

Personnel (September 1981):

Faculty:

S. Fung

R. Poe

Post-doctoral:

D. Beavis

D. Keane

Graduate Student:

A. Huie 
Institution: California Institute of Technology

Principal Investigator(s): F. Boehm

Contract No.: DE-ACO3-81ER00063

Titie:

"Research in Nuclear Physics at Low and Intermediate Energies, Neutrinos and X-Rays"

Address: $\quad$ Norman Bridge Laboratory of Physics

California Institute of Technology

Pasadena, CA 91125

Phone Number: $(213)-356-4 \angle 81$

Personnel (September 1981):

Faculty:

F. Boehm

A. Hahn

R. Powers

P. Vogel

J. Vuilleumier

Post-doctoral:

J. Gimlett

H. Kwon

D. Murray

Graduate Student:

A. Boothroyd

J. Markey 
Institution: Carnegie-Mellon University

Principal Investigator $(s)$ : P. Barnes, R. Eisenstein

Contract NO.: DE-ASO 3-81ERO3244

Title: "Experimental Medium Energy Nuclear Physics"

Address: Department of Physics

Carnegie-Melion University

Schenley Park

Pittsburgh, PA 15213

Phone Number: (412)-578-2745/2743

Personnel (September 1981):

Faculty:

P. Barnes

R. Eisenstein

W. Wharton

Post-doctoral:

B. Bassalleck

G. Franklin

P. Pile

\section{Graduate Student:}
R. Grace
C. Maher
R. Rieder
J. Szymanski 
Institution: Carnegie-Mellon University

Principal Investigator(s): M. Kaplan

Contract NO.: DE-ACO2-76ERI3246

Title: "Nuclear Research with Heavy Ions"

Address: Department of Chemistry Carnegie-Mellon University 4400 Fifth Avenue Pittsburch, PA 15213

Phone Number: (412)-578-3123

Personnel (September 1981):

Faculty:

M. Kaplan

Post-doctoral:

D. Eriksen

M. Kilair

D. Log̣an

Graduate Student:

D. Moses 
Institution:

Principal Investigator(s): P. Karol

Contract No.: DE-AC02-78ER04721

Title: "Experimental Nuclear and Radiochemistry: High-Energy Nuclear
Reactions".

Address: Department of Chemistry

Carnegie-Mellon University

4400 Fifth Avenue

Pittsburgh, PA 15213

Phone Number: (412)-578-3142

Personnel (September 1981):

Faculty:

P. Karol.

Post-doctoral:

S. Shibata

Graduate Student:

M. Tobin 
Institution: University of Chicago

Principal Investigator(s): A. Turkevich

Contract NO.: DE-ASO2-81ERI167

Title: "Nuclear Chemistry Research"

Address: $\quad$ Enrico Fermi Institute University of Chicago 5801 Ellis Avenue Chlcagu, IL 60637

Phone Number: (312)-733-8626

Personne1 (September 1981):

Faculty:

A. Turkevich

Post-doctoral:

$T$. Economov

H. Heydresser

K. Wiclgor

\section{Graduate Student:}

D. Boyce

J. Larosá 
Institution: Clark University

Principal Investigator(s): D. Brenner

Contract NO.: DE-ACO2-81ERI0493

Title: "Atomic Mass Measurements and Nuclear Spectroscopy at TRISTAN"

Address: $\quad$ Department of Chemistry

Clark University

Worcester, MA 01610

Phone Number: (617)-793-7114

Personnel (September 1981):

Faculty:

D. Brenner

Post-doctoral:

Graduate Student:

A. Aprahamian

M. Marte1 
Institution: Colorado School of Mines

Principal Investigator(s): F. Cecil

Contract NO.: DE-AC-02-80ERI0643

Title: "The Measurement of Total Cross Sections for Nuclear Reactions Induced by Low Energy Charged Part1cles"

Address: Department of Physics Colorado School of Mines Golden, LU BU401

Phone Number: (303)-279-0300

Personne) (September 1981):

Faculty:

F. Cecil

Post-doctoral:

Graduate Student:

R. Fahlsing 
Institution: University of Colorado

Principal Investigator(s): P. Kunz, E. Rost

Contract NO.: DE-ACO2-81ER40015

Title: "Theoretical Program"

Address: Nuclear Physics Laboratory

University of Colorado

Boulder; CO 80309

Phone Number: (303)-492-7483

Personnel (September 1981):

Faculty:

P. Kunz

E. Rost

Post-doctoral:

J. Shepard

E. Siciliano

\section{Graduate Student:}

H. Haghbin 
Institution: University of Colorado

Principal Investigator(s): D. Lind, R. Peterson

Contract NO.: DE-ACO2-81ER40014

Title: "Low Energy Experimental Nuclear Physics Research"

$\begin{array}{ll}\text { Address: } & \text { Nuclear Physics Laboratory } \\ & \text { University of Colorado } \\ & \text { Boulder, co } 80309\end{array}$

Phone Number: (303)-492-7483

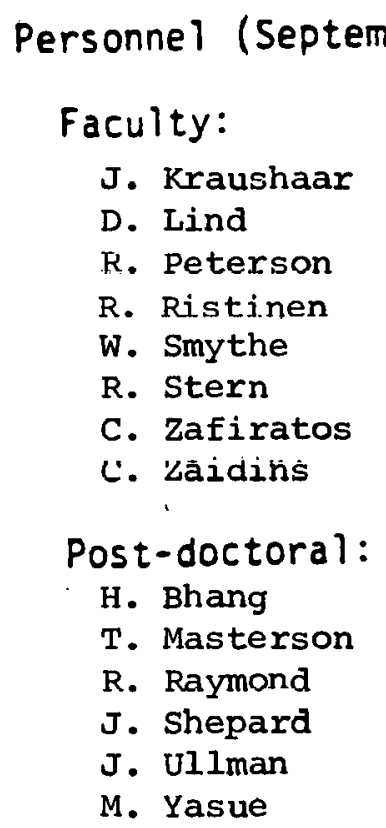

\section{Graduate Student:}
J. Benage
P. Craig
S. Dickey
J. Hamill
K. Hicks
R. Rodenburg 
Institution: University of colorado

Principal Investigator(s): R. Peterson

Contract NO.: DE-ACO2-81ER40016

Title: "Medium Energy Experimental Nuclear Physics Research"

Address: Nuclear Physics Laboratory

University of Colorado

Boulder, CO 80309

Phone Number: $(303)-492-7483$

Personnel (September 1981):

Faculty:

J. Kraushaar

D. Iind

R. Peterson

R. Ristinen

C. Zafiratos

Post-doctoral:

T. Masterson

J. Ullman

Graduate Student:

M. Rumore 
Institution: University of Connecticut

Principal Investigator(s): G. Rawitscher

Contract NO.: DE-ACO2-8OERO 4444

Title: "Nuclear Reaction Theory"

Address: Department of Physics Univerjity of Connectiout Storrs, CN 06268

Phone Number: (203)-486-4377

Personnel (September 1981):

Faculty:

G. Rawitscher

Post-doctora 1:

Graduate Student:

G. Rasonaivo 
Principal Investigator(s): D. Clark, G. Goldhaber

Contract No.: DE-AC02-81ER 10576

Title: "Experimental Studies of Nuclides Far from Stability"

Address: Nuclear Science and Engineering Program Cornell Unịversity

Ithaca, New York 14853

Phone Number: (607) 256-5224

Personnel (September 1981):

Faculty: D. Clark

G. Goldhaber

Post-doctoral:

T. R. Yeh

Graduate Student: 
Institution: Denison University

Principal Investigator(s): R. Winters

Contract NO.: DE-AC02-81ER02696

Title: "Determination of Neutron Cross Sections and Resonance Parameters"

$\begin{array}{ll}\text { Address: } & \text { Denison University } \\ & \text { Granville, OH } 43023\end{array}$

Phone Number: (611)-587-6327

Personnel (September 1981):

Faculty:

R. Winters

Post-doctoral:

Graduate Student: 
Institution: Duke University

Principal Investigator(s): E. Bilpuch

Contract NO.: DE-ACO5-81ER01067

Title: "Studies of Nuclear Structure using Neutrons and Charged Particles"

$\begin{array}{ll}\text { Address: } & \text { Triangle Universities Nuclear Laboratory } \\ & \text { Duke University } \\ \text { Duke Station, } \\ \text { Durham, NC } 27706\end{array}$

Phone Number: (919)-684-8111

Personnel (September 1981):

Faculty:

E. BIipuch

H. Lewis

F. Purser

N. Roberson

R. Walter

H. Weller

\section{Post-doctoral:}
R. Byrd
G. Tunqate
C. Westerfe:Idt

\section{Graduate Student:}
R. August
G. Mitev
M. Bonyata
K. Murphy
C. Fitzpatrick
R. Nelson
C. Floyd
R. Pedroni
P. Guss
J. Shriner
G. Honore
D. Wagonaar
C. Howell
B. Warthen
s. King
K. Whatley
s. Manglos
M. Wright 
Laboratory: EG\&G Idaho, Inc.

Division: Physics

Personnel (September 1981):

Permanent Staff:
R. Bunting
G. Caffrey
R. Greenwood
R. Heath ${ }^{*}$
R. Helmer
M. Lee
C. Reich

Post-doctorals and Visitors:

H. Willmes

Graduate Students:

R. Madigan
Address: Idaho National Engineering Lab EG\&G Idaho, Inc. P. 0. Box 1625 Idaho. Fal1s, ID 83401

Phone: (208) 526-1351

FTS 583-1291 (town)

FTS 583-44.47 (site)

*Person in charge 
Principal Investigator(s): D. Robson

Contract NO.: DE-ASO5-81ER10419

Title: "Research in Theoretical Nuclear Physics"

Address: Department of Physics

Florida State University

Tallahassee, FL 32306

Phone Number: (904)-644-1429

Personnel (September 1981):

Faculty:

D. Robson

Post-doctoral:

C. Long

D. Stanley

\section{Graduate Student:}

R. Campbell

W. Jaronski. 
Institution: Georgia Institute of Technology

Principal Investigator(s): R. Fink

Contract No.: DE-ASO5-76ERO3346

Title:

"Nuclear Chemistry Research and Spectroscopy with Radioacitve Sources"

Address: $\quad$ Department of Chemistry

Georgia Institute of Technology

Atlanta, GA 30332

Phone Number: (404)-894-4030

Personnel (September 1981):

Faculty:

R. Fink

Post-doctoral:

R. Braga

Graduate Student:

B. Gnade

P. Semmes 
Institution: Georgia Institute of Technology

Principal Investigator(s): J. Wood

Contract NO.: DE-ASO5-81ER10599

Title: "Nuclear Structure from Radioactive Decay"

Address: School of Physics

Georgia Institute of Technology

Atlanta, GA $\quad 30332$

Phone Number: (404)-894-5262

Personnel (September 1981):

Faculty:

J. Wood

Post-doctoral:

Graduate Student:

C. Papanicolopulos 
Institution: Georgia State University

Principal Investigator(s): G. Petitt

Contract NO.: $\quad$ DE-ASO5-80ER10418

Title: "Light Particle Emission Studies in Heavy Ion Nuclear Reactions"

Address: $\quad$ Department of Physics \& Astronomy Georgia State University

Atlanta, GA 30303

Phone Number: $(404)=$ ก.5ร-3221

Personnel (September 1981):

Faculty:

G. Petitt

Post-doctoral:

Graduate Student: 
Institution: George Washington University

Principal Investigator(s): E. Harper, D. Lehman, F. Prats

Contract No.: DE-AS05-79ER10332

Title: $\quad$ "Investigations of the Structure and Electromagnetic Interactions of Few-Body Systems"

Address: $\quad$ Department of Physics George Washington University

washington, DC 20052

Phone Number: (202)-676-6846

Personne1 (September 1981):

Faculty:

E. Harper

D. Lehman

F. Prats

Post-doctoral:

Graduate Student: 
Institution: Harvard University

Principal Investigator $(s): \quad R$. Wilson

Contract NO.: DE-AS02-80ER10621

Title: "Study of parity Violation in Electron-Carbon Scattering"

Address: The Physics Laboratories Harvard University

Cambridge, MA 02138

Phone Number: (617)-495-338\%

Personnel (September 1981):

Faculty:

M. Fiering

$\mathrm{J}$. Harrington

H. Thomas, Jr.

R. Wilson

Post-doctoral:

E. Crouch

J. Murray

Graduate Student:

R. Ellgas 
Institution: University of Houston

Principal Investigator(s): B. Mayes, E. Hungerford

Contract NO.: DE-ASO5-76ER03948

Title: "Hypernuclear Spectroscopy"

Address: $\quad$ Central Campus Department of Physics University of Houston Houston, TX 77004

Phone Number: (713)-749-2832

Personne1 (September 1981):

Faculty:

E. Hungerford

B. Mayes

L. Pinsky

Post-doctoral:

A. Hancock

H. Piekarz

K. Sekharan

Graduate Student:

S. Bart

K. Forester

R. Hackenberg 


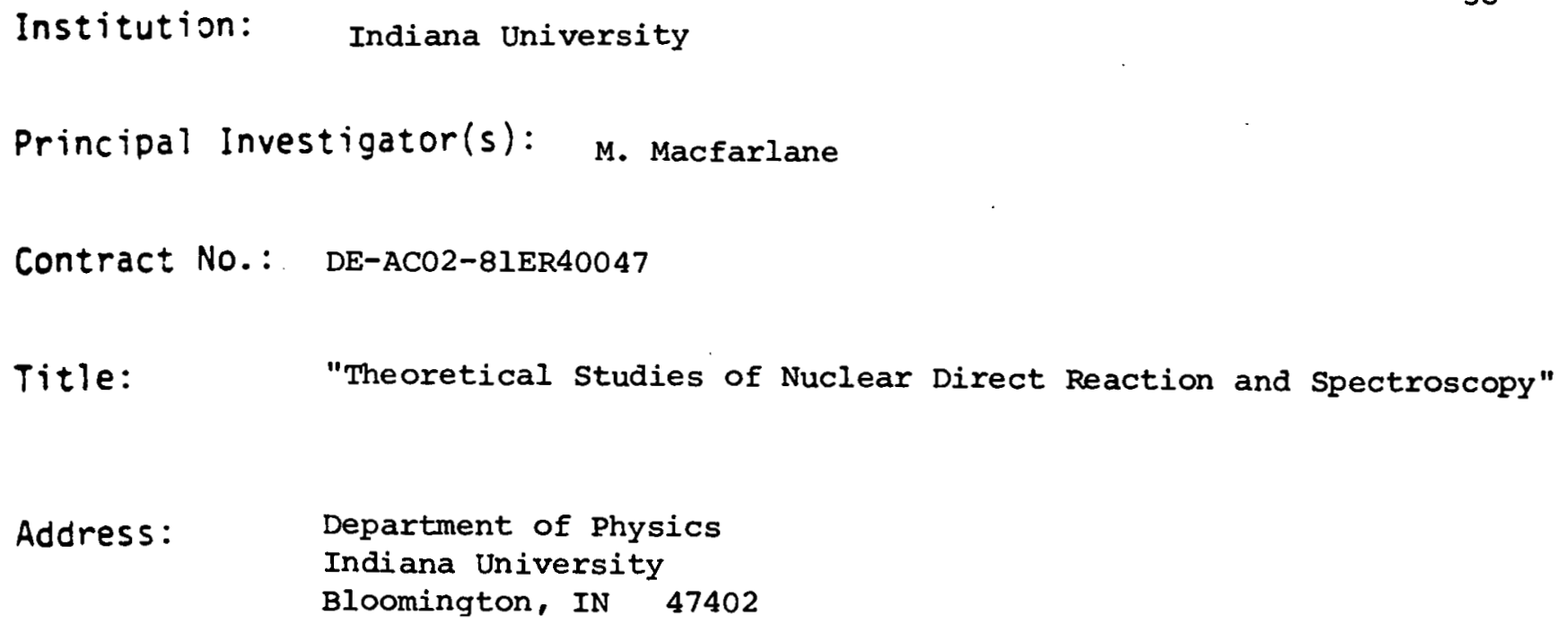

Phone Number: (012)-337-3709

Personnel (September 1981):

Faculty:

M. Macfarlane

Post-doctoral:

T. Cooper

\section{Graduate Student:}

M. Lambert 
Institution: University of Indiana

Principal Investigator(s): V. Viola

Contract No.: $\quad$ DE-AS02-81ER40007

Title: "Studies of Heavy-Ion-Induced Nuclear Reactions"

Address: $\quad: \quad$ Department of Chemistry

University of Indiana

Chemistry Building

Bloomington, IN 47405

Phone Number: (812)-337-6537

Personnel (September 1981):

Faculty:

V. Viola, Jr.

\section{Post-doctoral:}

K. Kwiatkowski

L. Woo

S. Zhou

\section{Graduate Student:}

L. Nowick

M. Walker 
Institution: Iowa State University

Principal Investigator $(s):$ D. Lewiis

Contract NO.: DE-AS02-81ER10496

Title: "Sizes and Shapes of Short-Lived Nuclei via Laser Spectroscopy"
Address: Department of Physics Iowa State University
12 Physics Building
Ames, Iowa 50011

Phone Number: (515)-294-5440

FTS $865-5440$

Personnel (September 1981):

Faculty:

D. Lewis

Post-doctoral:

Graduate Student:

R. Evans 
Institution: University of Iowa

Principal Investigator(s): W. Klink, G. Payne

Contract NO.: DE-ACO2-81ER40038

Title: "Configuration Space Faddeev Calculations"

Address: Department of Physics and Astronomy University of Iowa

Iowa City, Iowa $\mathbf{5 2 2 4 2}$

Phone Number: (319)-353-4343

Personnel (September 1981):

Faculty:

W. Kłink

G. Payne

Post-doctoral:

Graduate Student:

R. Downie 
Institution: John Hopkins University

Principal Investigator(s): L. Madansky, Y. Lee

Contract No.: $\mathrm{DE}-\mathrm{ACO} 2-76 \mathrm{ERO} 3274$

Title: "Nuclear Moments and Nuclear Structure"

$\begin{array}{ll}\text { Address: } & \text { Department of Physics } \\ \text { Johns Hopkins University } \\ \text { Homewood Campus } \\ \text { Baltimore, MD } 21218\end{array}$

Phone Number: (301)-338-7354

Personnel (September 1981):

Faculty:

Y. Lee

L. Madansky

J. Walker

Post-doctoral:

T. Hallman

E. McIntyre

Graduate Student:

R. Levin 
Institution: University of Kansas

Principal Investigator(s): F. Prosser

Contract NO.: DE-ACO2-8BER10420

Title: "Fusion Measurements in Light and Medium Mass Heavy Ion Reactions"

Address: Department of Physics and Astronomy University of Kansas

Lawrence, Kansas 66045

Phone Number: (913) 864-4741

Personnel (September 1981):

Faculty:

F. Prosser

Post-doctoral:

Graduate Student:

R. Racca 
Institution: University of Kentucky

Principal Investigator(s): F. Gabbard, R. Hershberger

Contract No.: DE-AS05-81ER10642

Title: "Total $(p, n)$ and $(\alpha, n)$ Cross Sections for Nucleosynthes is and Nuclear Applications"

Address: Department of Physics \& Astronomy University of Kentucky

Lexington, KY 40506

Phone Number: (606)-258-2960

Personnel (September 1981):

Faculty:

F. Gabbard

R. Hershberger

Post-doctoral:

B. Fuqua

G. Pepper

Graduate Student:

M. Smith 
Laboratory: Lawrence Berkeley Laboratory

Division: Nuclear Science

Accelerator and Fusion Research

Personne1 (September 1981):
Address: Lawrence Berkeley Lab

I Cyclotron BIvd.

Berkeley, CA 94720

Phone: (415) 486-4000

Permanent Staff:
A. Alonso
B. Gavin
C. Leemann
J. Rasmussen
E. Browne-Moreno
A. Ghiorso
C. Lyneis
L. Schroeder
J. Cerny*
N. Glendenning
C. Maples, Jr.
G. Seaborg
0 Chamberlain
R. Gough
L. Moretto
F. Seiph
W. Chu
D. Greiner
R. Muller
J. Staples
D. Clark
C. Gruhn
W. Myers
H. Steiner
H. Conzett
H. Grunder*
S. Nagamiya
F. Stephens
K. Crebbin
M. Gyulassy
M. Neiman
J. Stevenson
T. Criswell
B. Harvey
J. Nitschke
R. Stokstad
K. Crowe
H. Heckman
M. Nurmia
W. Swiatecki
J. Dairiki
D. Hendrie
V. Perez-Mendez
$T$. Symons
M. Deleplanque
J. Kalnins
A. Poskanzer
P. Tans
R. Diamond
H. Kim
P. Price
G. Wozniak
R. Firestone
K. Kim
E. Friedlander
B. Leemann
H. Pugh
M. Zisman
J. Randrup

Post-doctorals and Visitors:
J. Bistirlich
R. Koontz
T. Renner
A. Shor
J. Boguta
R. McDonald
H. Riedesel
I. Tserruya
G. Fai
E. Moeller
H. Ruck
R. Wada
K. Frankel
T. Mulera
V. Ruck
A. Warwick
M. Murphy
A. Sagle

Graduate Students:
S. Abachi
C. Clawson
Y. Morita
J. Sullivan
C. Albiston
K. Ganezer
D. Murphy
R. Treuhaft
A. Bice
R. McFarland
J. Musser
R. Welch
M. Budiansky
P. McGaughey
R. Parry
J. Wouters
M. Cable
J. Molitoris
L. Sobotka
S. Yashita
S. Chessin
K. Moody
L. Somerville
W. Zajc
F. Zarbakhsh 
Laboratory: Lawrence Livermore Laboratory.

Division: Physics Chemistry

Personne1 (September 1981):

Permanent Staff:
B. Berman
R. Borg
R. Hâight
D. Letch
M. Lindner
R. Meyer
H. Meldner

Address: Lawrence Livermore Lab P. 0. Box 808 Livermore, CA 94550

Phone: (415) 422-1100 FTS $532-1100$ 
Laboratory: Los Alamos National Laboratory

Division: MP-Divison

Personnel (September 1981):

Permanent Staff:
L. Agnew
D. Cochran
C. Hoffman
J. Allred
M. Cooper
J. Amann
R. Damjanovich
E. Hoffman
J. Hurd
H. Baer
R. DeHaven
J. Donahue
J. Jarmer.
P. Berardo
D. Doss
J. Bergstein
J. Frank
R. Boudrie
P. Gram
J. Bowman
D. Grisham
J. Bradbury
D. Hagerman
A. Browman
C. Hansen
R. Brown
J. Harrison
S. Brown
v. Hart
R. Burman
R. Heffner
M. Hoehn
H. Butler
E. Chamberlin
T. Putnam
L. Rosen
V. Sandberg
G. Sanders
S. Schaller
M. Schillaci
D. Simmonds
R. Stevens
G. Swain
N. Tanaka
H. Theissen
0. Van Dyck
J. Wallace
R. Werbeck
R. Williams
R. York

Address: Los Alamos National Lab University of California P. 0. Box:7.663 Los Alamos, NM 87545

Phone: (505) 667-5061 FTS 843-5061

Post-doctorals and Visitors:
C. Boekema
W. Cornetius
W. Kinnison
R. Talaga
D. Brenner
G. Hogan
M. Leitch
V. Yuan
R. Carlini.
M. Hynes
H. Matis
H. Ziock

Graduate Students:
M. Barlett
S. Elston
C. Harvey
E. Milner
G. Blanpied
R. Fergerson
D. Holtkamp
S. Nanda
W. Cottingame
S. Greene
J. Marshall
B. Nelson
P. Seidl

*Person in charge 
Laboratory: Los Alamos National Laboratory

Division: P-Division

Personnel (September 1981):

Permanent Staff:

H. Anderson

B. Bonner

H. Britt

R. Brown ${ }^{*}$

J. Browne

R. DeVries

N. Digiacomo

D. Drake

N. Jarmie

M. Mejer

J. Moss

G. Russe11

B. Shera

J. Simmons

J. Sunier

S. Wender

D. Wohlfahrt

Post-doctoral and Visitors:

J. Vander Plicht
Address: Los Alamos National Lab P.1:0. Box 1663 Los Almos, NM 87545

Phone: (505) 667-5061

FTS 843-5061

*Person in charge 
Laboratory: Los Alamos National Laboratory

Division: AT Division

Personnel (September 1981):

Permanent Staff:
R. Hoberling
R. Jameson
E. Knapp
R. Martin
J. Potter
D. Reid
R. Stokes
D. Swenson
P. Tallerico
L. Young

Address: Los Alamos National Lab P. 0. Box 1663 Los Alamos; NM 87545

Phone: (505) 667-5061

FTS 843-5061

*Person in charge 
Laboratory: Los Alamos National Laboratory

Division: Chemistry--Nuclear Chemistry

Personne1 (September 1981):

Permanent Staff:
G. Butler
B. Dropesky
G. Giesler
D. Hoffman *

Address: Los Alamos National Lab P. 0. Box 1663 Los Alamos, NM 87545

Phone: (505) 667-5061

FTS 843-5061
1. Kni ghil:
L. Liu
C. Orth
G. Vieira
J. Wilhelmy

Post-doctoral and Visitors;
J. Clark
J. Gilbert
D. Long 
Laboratory: Los Alamos National Laboratory

Division: Theory

Personnel (September 1981):

Permanent Staff:

E. Arthur

D. Dodder

T. England

J. Friar

B. Gibson

W. Gibbs

J. Ginocchio

T. Goldman

G. Hale

L. Heller

P. Herczeg

R. Silbar

G. Stephenson *
Address: Los Alamos National Lab P. 0. Box 1663 Los Alamos, NM 87545

Phone: (505)-667-5061

FTS 843-5061

Post-doctorals and Visitors:

A. Aerts

A. Cass

W. Haxton

P. Muiders

E. Tomusiak

*Person in charge 
Institution: Louisiana State University

Principal Investigator(s): P. Kirk

Contract NO.: DE-AS05-8IER04699

Title: "Experiments using the TASS and HISS Spectrometers at the Bevalac"

Address: Department of Physics and Astronomy Louisiana State University

Baton Rouge, LA 70803

Phone Number: (504)-388-5849

Personnel (September 1981):

Faculty:

P. Kirk

Post-doctoral:

- G. Krebs

\section{Graduate Student:}

$J$. Engelage 
Institution: Louisiana State University

Principal Investigator(s): E. Zganjar

Contract NO.: DE-ASO5-81ER04935

Title: "The Structure of Nuclei Far From Beta Stability"

Address: Department of Physics Louisiana State University Baton Rouge, IA 70803

Phone Number: (FTS) $-688-8400$ Commercial 504-388-8400

Personnel (September 1981):

Faculty:

J. Draayer

E. Zganjar

Post-doctoral:

J. Cole

Graduate Student: 
Institution: University of Lowell

Principal Investigator(s): L. Beghian, G. Kegel

Contract No.: $\quad$ DE-ACO2-79ER10344

Title: "Neutron Scattering Studies in Thorium and Uranium"

Address: $\quad$ Department of Physics University of Lowell

$\perp$ University Avenue

Lowell, MA 01054

Phnne Numberr: $\quad(617)-452-5000$

Personnel (September 1981):

Faculty:

J. Egan

G. Kegel

D. Pullen

Post-doctoral:

Graduate Student:

C. Ciarcia

G. Goswami

J. Ring

J. Shao

P. Surette 
Institution: University of Maryland

Principal Investigator(s): A. Mignerey

Contract NO.: DE-ASO5-76ER05172

Title: "Reaction Mechanism Studies of Heavy-Ion-Induced Nuclear Reactions"

Address: Department of Chemistry University of Maryland College Park, MD 20742

Phone Number: (301)-454-4422

Personnel (September 1981):

Faculty:

A. Mignerey

Post-doctoral:

S. Barkan

A. Cokmon

\section{Graduate Student:}

S. Merouane

F. Moghadami 
Institution: University of Maryland

Principal Investigator(s): E. Redish

Contract NO.: DE-ASO5-76ER05126

Title: "Theoretical Nuclear Physics Calculations"

Address: Department of Physics \& Astronomy University of Maryland

Collége tarkk, ML $20 / 42$

Phone Number: (301)-454-3531.

Personnel (September 1981):

Faculty:

M. Banerjee

M. Dworzecka

J. Griffin

W. MacDonald

E. Redish

J. Van Orden

5. Wallace

Post-doctoral:

M. Birse

A. Blin

D. Giebink

B. Hiller

V. Mandelzweig

D. Schneider

K. Stricker-Bauer

\section{Graduate Student:}
J. Adams
G. Kim
R. Perry
F. Pineda
R. Smith
C. Yannouleas 
Institution: University of Maryland

Principal Investigator $(s):$ w. Walters

Contract No.: DE-AS05-81ER10494

Title: "Radioactive Decay Studies at TRISTAN"

Address: Department of Chemistry University of Maryland College Park, MD 20742

Phone Number: (301)-454-4421

Personnel (September 1981):

Faculty:

w. Wälters

Post-doctoral:

c. Chung

$x$

Graduate Student:

s. Failier

C. Stone 
Institution: Massachusetts Institute of Technology

Principal Investigator $(s)$ : P. Demos

Contract No.: DE-AC02-76ER03069

Title: "Bates Linear Accelerator".

Address: Datcs Linear Acsel.erator

P. O. Box 95

Middleton, MA 01949

Phone Number: (617)-245-6600

Personnel (September 1981):

Faculty: Permanent Staff
W. Barber
R. Redwine
A. Bernstein
W. Sapp
W. Bertozzi
C. Sargent
K. Blomquist
W. Turchinetz
P. Demos
C. Williams on
M. Deutsch
j. Flanz
S. Kowalski
J. Matthews
Post-doctoral:
G. Adams
M. Ruims
S. Dytman
M. Finn
D. Kelly
M. Kovaeh
T. Scharter

\section{Graduate Student:}
A. Barber
R. Miskimen
W. Burger
B. Murdock
J. Comuzzi
J. Nelson
B. Cottman
B. Pugh
B. Craft
B. Quinn
S. Evangelides
M. Schultze
M. Farkondeh
R. Schumacher
W. Hersman
S. Turley
C. Hyde
J. Wong
c. Lin
s. Wood 
Institution: Massachusetts Institute of Technology

Principal Investigator(s): A. Kerman

Contract No.: DE-AC02-76ER03069

Title: "Nuclear Theory"

Address: Laboratory for Nuclear Science

Massachusetts Institute of Technology

Cambridge, MA 02139

Phone Number: (617)-253-7072

Personne1 (September 1981):

Faculty:

M. Baranger

H. Feshbach

A. Kerman

S. Levit

E. Loman

E. Moniz

J. Negele

F. Villars

Post-doctoral:

K. Ohta

M. Robc1

H. Tang

G. Vichniac

Graduate Student:

B. Karaoglu

T. Karapiperis

D. Root

R. Sinha

T. Troudet

$\mathrm{J}$. Weinstein 
Institution: Massachusetts Institute of Technology

Principal Investigator(s): E. Cosman

Contract No.: DE-AC02-76ER03069

Title: "Heavy Ion Nuclear Physics Research"

Address: Laboratory for Nuclear Science Massachusetts Institute of Technology Cambridge, MA 02139

Phone Number: 617-253-4153

Personnel (September 1981):

Faculty:

E. Cosman

H. Enge

L. Grodzins

S. Steadman

Post-doctoral:

M. Beckerman

M. Bechara

D. Branford

Graduate Student:

R. Boisseau

S. Gazes

D. I1ya

A. Kofahi

R. Ledoux

C. Ordonez

A. Smith 
Institution: University of Massachusetts

Principal Investigator(s): G. Peterson

Contract No.: DE-ACO2-76ER02853

Title: "Nuclear Physics Research"

Address: Department of Physics \& Astronomy University of Massachusetts Amherst, MA 01003

Phone Number: (303)-492-7483

Personnel (September 1981):

Faculty:

J. Dubach

R. Hicks

R. Lindgren

G. Peterson

Post-doctoral:

A. Hotta

Graduate Student:
$R$. Huffman
B. Parker
M. Plum 
Institution: Michigan State University

Principal Investigator(s): H. Blosser

Contract NO.: DE-ASO2-80ERI7605

Title: "National Superconducting Gyclotron Laboratory"

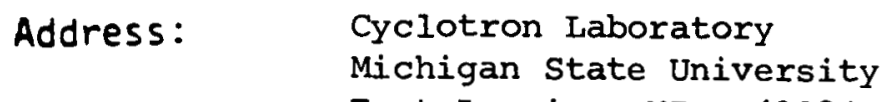

East Lansing, MI 48824

Phone Number: (517)-355-9671

FTS 375-9671

Personnel (September 1981):

Faculty:

H. Blosser

M. Gordon

B. Waldman

Post-doctoral:

Graduate Student: 
Institution: Michigan State University

Principal Investigator(s): P. Signeli

Contract NO.: DE-AC02-81ERI0516

Title: "Nucleon-Nucleon Theory and Phenomenology"

Address: $\quad$ Department of Physics Michigan State University East Lansing, MI 48824

Phone Number: (517)-355-9665

FTS 375-9665

Personne1 (September 1981):

Faculty:

F. Freiheit

P. Signell

Post-doctoral :

G. Kales

\section{Graduate Student:}

M. brandl 
Institution: University of Michigan

Principal Investigator(s): G. Knoll

Contract NO.: DE-ASO2-81ER02025

Title: "Absolute Measurements of Neutron Cross Sections"

Address: Department of Nuclear Engineering University of Michigan

Cooley Building, North Campus

Ann Arbor, MI 48109

Phone Number: (313)-764-4260

Personnel (September 1981):

Faculty:

G. Knoll

Post-doctoral:

Graduate Student:

$J$. Hassberger

M. Mahdavi $i$

K. Zasadny 
Institution: Midale Tennessee State

Principal Investigator(s): R. Carlton

Contract NO.: DE-ASO5-8OERIOT10

Title: "Nuclear Structure Studies via Neutron Interactions"

Address: Department of Chemistry \& Physics Middle Tennessee State University Murfreesboro, TN 37132

Phone Number: (615)-898-2130

Personnel (September 1981):

Faculty:

R. Carlton

Post-doctoral:

Groduate Student: 
Institution: University of Minnesota

Principal Investigator $(\mathrm{S})$ : B. Bayman, P. E11is, Y. Tang

Contract No.: DE-AC02-79ER10364

Title: "Research in Theoretical Nuclear Physics"

Address: $\quad$ School of Physics and Astronomy

Tace Labuiatory of Phyoico

University of Minnesota

116 Church Street 8.E.

Minneapolis, MN bל4b5

Phone Number: (612)-373-0240

Personnel (September 1981):

Faculty:

B. Bayman

P. ElIis

Y. Tang

Post-doctoral:

S. Chakravarti

Y. Fujiwara

\section{Graduate Student:}

Z. E1-Itaoui

P. Shen 
Institution: University of Minnesota

Principal Investigator(s): D. Dehnhard

Contract NO.: DE-ACO2-79ER10423

Title: "Nuclear Structure Studies with Pions and Heavy Ions"

Address: Department of Physics

Minnesota University

Minneapolis, MN 55455

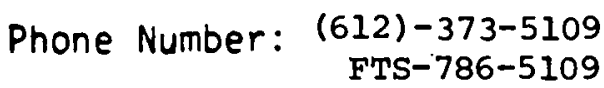

Personnel (September 1981):

Faculty:

D. Dehnhard

Post-doctoral:

D. Holtkamp

V. Shkolnik

Graduate Student: 
Institution: University of Minnesota

Principal Investigator(s): N. Hintz

Contract NO.: DE-ASO5-81ER04215

Title: "Nuclear Structure Studies at Intermediate Energles"

Address: School of Physics and Astronomy University of Minnesota 148 Phyeios Building Minneapol1s, Minesula 5J45J

Phone Number: (612)-373-3066

Personnel (September 1981):

Faculty:

N. Hintz

Post-doctoral:

M. Franey

M. Gazzaly.

Graduate Student:

D. Cook 
Laboratory: National Bureau of Standards

Division: Radiation Source and Instrumentation

Personnel (September 1981):

Permanent Staff:

P. Debenham

S. Penner *

M. Wilson

Post-doctoral and Visitors:

N. Yoder
Address: National Bureau of Standards Washington, DC 20234

Phone: (301) 921-1000 
Laboratory: National Bureau of Standards

Division: Nuclear Radiation

Personnel (September 1981):

Permanent Staff:

\section{Bowman}

A. Carlson

R. Caswe $11^{\star}$

J. Grundl

A. Johnson

C. Kuyatt

E. Schroder.

A. Wasson
Address: National Bureau of Standards Washington, DC 20234

Phone: (301) 921-2652 
Institution: University of New Hampshire

Principal Investigator(s): J. Heisenberg

Contract No.: DE-AS02-79ER10338

Title: "Nuclear Structure Investigations on Spherical Nuclei"

Address: $\quad$ Department of Physics

University of New Hampshire

DeMeritt Ha11

Durham, NH 03824 .

Phone Number: (602)-862-1950

Personnel (September 1981):

Faculty:

J. Dawson

J. Heisenberg

R. Lambert

Post-doctoral:

0 . Schwentker

\section{Graduate Student:}


Institution: New Mexico State University

Principal Investigator(s): G. Burleson

Contract No.: DE-AS04-81ER03591

Title: "Experimental Studies of Various Nucleon and Pion Interactions at Intermediate Energies"

Address: Department of Physics New Mexico State University

Las Cruces, NM 88003

Phone Number: (505)-646-3831

Personnel (September 1981):

Faculty:

G. Burleson

Post-doctoral:

w. Cottingame

S. Greene

Graduate Student:

$\checkmark$ 
Institution: University of New Mexico

Principal Investigator(s): J. Robertson

Contract NO.: DE-ASO4-79ERI04II

Title: "Neutron Diagnostics of Fusion Systems and Related Cross Section Measurements"

Address: Department of Chemical and Nuclear Engineering University of New Mexico

Albuquerque, NM 87131

Phone Number: (505)-277-2209

Personnel (September 1981):

Faculty:

J. Robertson

Post-doctoral:

Graduate Student:

A. Arbildo

D. Sloan 
Institution: University of New Mexico

Principal Investigator(S): D. Wolfe

Contract NO.: DE-ASO4-80ERO3347

Title: "Nuclear Physics Studies at Intermediate Energies"

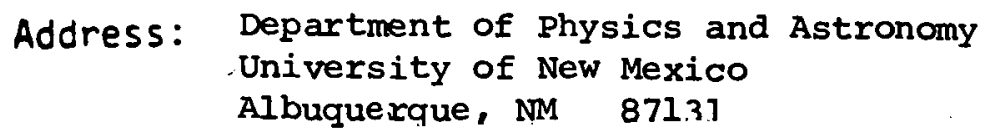

Phone Number: (505)-277-2616

Personnel (September 1981):

Faculty:

B. Dieterle

C. Leavitt

D. Wolfe

Post-doctoral:

Graduate Student:

Denes

Herman 
Institution: State University of New York at Albany

Principal Investigator(s): J. Garg

Contract NO.: DE-ACO2-76ER02439

Title:

"Measurement and Analysis of Neutron Cross Bections with the Oak
Ridge Linear Accelerator ORELA Neutron Facility"

Address: Nuclear Accelerator Laboratory State University of New York 1400 Washington Avenue

Albany, NY 12222

Phone Number: (518)-457-8450

Personnel (September 1981):

Faculty:

J. Garg

Post-doctoral:

V. Tikku

Graduate Student: 
Institution: State University of New York at stony Brook

Principal Investigator(s): J. Alexander

Contract NO.: DE-ASO2-81ERO 3470

Title: "Nuclear Reaction Studies"

Address: Department of Chemistry

State University of New York

Stony Drook, NY 11794

Phone Number: (516)-246-5055

Personnel (September 1981):

Faculty:

J. Alexander

Post-doctoral:

D. Guerreau

L. Vaz

Graduate Student:

E. Duek

R. Lacy 
Institution: State University of New York, Stony Brook

Principal Investigator(s): G. Brown

Contract NO.: DE-ACO2-81ERI3001

Title: "Research in Theoretical Nuclear Physics"

Address: Physics Department State University of New York Stony Brook, NY 11794

Phone Number: (516)-246-7955

Personnel (September 1981):

Faculty:

G. Brown

L. Castillejo

A. Jackson

T. Ku申

S. Lee

T. Mazurek

A. Yahill

Post-doctoral:

J. Applegate

w. Ferchlander

J. Jun

E. Krotscheck

F. Osterfield

R. Smith

J. Wambach

Graduate Student: 
Institution: City University of New York, Brooklyn College

Principal Investigator $(s):$ H. Lancman

Contract NO.: DE-ASO2-81ERI0452

Title: "High Resolution Photofission Measurements in ${ }^{238} \mathrm{U}$ and ${ }^{232}$ Th"
Address: Department of Physics Gity Iniversity of New York Bedford Avenue and Avenue $\mathrm{H}$ Brooklynn, NY 11210

Phone Number: (212) $780-5810$

Personnel (September 1981):

Faculty:

H. Lancman

Post-doctoral:

M. Ismael

Graduate Student:

H. Zhang

A. Khazbak 
Institution: City University of New York, Brooklyn College

Principal Investigator $(s): \quad$ c. Trail

Contract No.: DE-ACO2-81ER40023

Title:

"Bremsstrahlung from Nuclear Scattering at Low Energy Near
a Resonance"

Address: Department of Physics

City University of New York

Brooklyn, NY 11210

Phone Number: (212)-780-5811/5418

Personnel (September 1981):

Faculty:

P. Lesser

M. Liou

C. Trail

Post-doctoral:

Graduate Student: 
Institution: City University of New York, City College

Principal Investigator(s): M. Lubell

Contract NO.: DE-ACO2-81ER40017

Title: "Parity Violating Weak Neutral Current Effects in Elastic $e^{l .2}$ C scattering"

Address: Department of Physics

City College of CUNY

138th Street \& Convent Avenue

New York, NY 10031

Phone Number: (212)-690-8316

Personnel (September 1981):

Faculty:

M. Lubell

Post-doctoral:

Graduate Student: 
Institution: New York University

Principal Investigator(s): M. Kalos

Contract NO.: DE-ACO2-81ERI0353

Title: "Computational Methods for the Nuclear and Neutron Matter Problems"

$\begin{array}{ll}\text { Address: } & \text { Courant Institute of Mathematical Sciences } \\ & \text { New York University } \\ 251 \text { Mercer Street } \\ \text { New York, NY } 10012\end{array}$

Phone Number: (212)-460-7100

Personnel (September 1981):

Faculty:

M. Kalos

Post-doctoral:

K. Schmidt

Graduate Student:

K. Mohanty 
Institution: North Carolina State University

Principal Investigator $(s):$ s. Cotanch

Contract NO.: DE-ASO5-79ERI0407

Title: "Study of the Interaction of Kaons and Photons with the Atomic Nucleus"

Address: Department of Physics North Carolina State University BOx 5342

Raleigh, NC 27550

Phone Number: (919)-737-2512

Personnel (September 1981):

Faculty:

s. Cotanch

Post-doctoral:

\section{Graduate Student:}

J. Lafferty 
Institution: North Carolina State University

Principal Investigator(s): L. Seagondollar

Contract NO.: DE-AS05-81ER0 3624

Title: "Nucleai Structure Research at Triangle Universities Nuclear Laboratory (TUNL)"

Address: Physical \& Mathematical Sciences North Carolina State University Raleigh, NC 27650

Phone Number: (919)-737-2525

Personnel (September 1981):

Faculty:

L. Seagondollar

G. Mitchell

D. Tilley

C. Gould

Post-doctoral:

J. Dave

\section{Graduate Student:}

P. Ramakrishman

L. Ward 
Institution: University of North Carolina at Chapel Hill

Principal Investigator(s): E. Merzbacher

Contract NO.: DE-AS05-81ER02408

Title: . "Studies of Nuclear Processes"

Address: Department of Physics and Astronomy University of North Carolina Chapcl IIill, NC $\cdot 27514$

Phone Number: (919)-933-2079

Personnel (September 1981):

Faculty:

R. Anderson

T. Clegg

E. Ludwig

E. Merzbacher

W. Thompson

Post-doctoral:

Y. Aoki

D. Kopf

F. Xma

\section{Graduate Student:}

B. Burks

R. Varner

J. Wilkerson 
Institution: Northwestern University

Principal Investigator(s): K. Seth

Contract NO.: DE-ACO2-81ERO2706

Title: "Nuclear Structure Studies with Medium Energy Probes"

Addres5: Department of Physics \& Astronomy Northwestern University

Evanston, IL 60201

Phone Number: (312)-492-3123/3644

Personnel (September 1981):

Faculty:

K. Seth

Post-doctoral:

D. Kielczewka

A. Saha

D. Smith

Graduate Student:

D. Barlow

M. Kaletka 
Institution: Oak Ridge Associated Universities

Principal Investigator(s): E. Spejewski

Contract No.: DE-AC05-760R00033

Title: "University Isotope. Separator-Oak Ridge"

Address: Holifield Heavy-Ion Research Facility

nak Ridge Associated Universities

Oak Ridge, TN 37830

Phone Number: (6.15)-62/-1/740

Personnel (September 1981):

Faculty:

H. Carter

G. Leander

R. Mlekodaj

E. Spejewsk1

Post-doctoral:

Graduate Student: 
Laboratory: Oak Ridge National Laboratory Division: Physics

Personnel (September 1981):

Permanent Staff:
G. Alton
D. Hensley
S. Mosko
R. Auble
D. Hetrick
F. Obenshain
J. Ball
N. Hill
F. Perey
J. Beene
D. Horen
F. Plasil
Z. Bell
E. Hudson
S. Raman
C. Bemis
S. Hurst.
R. Robinson
J. Benjamin
C. Johnson
G. Satchler
$F$. Bertrand
J. Johnson
R. Sayer
J. Biggerstaff
N. Johnson
M. Schmorak
J. Blankenship
C. Jones
D. Shapira
J. Dabbs
R. Juras
G. Slaughter
K. Davies
I. Lee
R. Spencer
J. Dickens
D. Larson
P. Stelson *
Y. Ellis-Akovali
T. Lewis
H. Todd
K. Erb
R. Lord
J. Todd
R. Ferguson
C. Ludemann
K. Toth
J. C. Ford
R. Macklin
C. Vane
C. $\mathrm{Fu}$
J. Mann
T. Welton
C. Fulmer
J. Martin
L. Weston
J. Gomez del Campo
M. Martin
C. Wong
E. Gross
J. McConnell
M. Halbert
F. McGowan
G. Young
B. Harmatz
J. McGrory
J. Harvey
W. Milner

Post-doctorals and Visitors:
M. Fewell
R. Novotny
T. Sjoreen
D. Moltz
M. Rhoades-Brown

Graduate Students:

D. Digregorio

P. 0. Box $X$

Oak Ridge, TN 37830

(615) $576-5454$

FTS $624-4415$ 
Institution: Ohio University

Principal Investigator(s): R. Lane

Contract No.: DE-ACO2-81ER02490

Title: "Isospin and Giant Resonance Effects in Neutron Scattering and Neutron Induced Charged Particle Reactions"

Address: $\quad$ Department of Physics

Ohio University

Accelerator Laboratory

Athens, OH 45701

Phone Number: (614)-594-6970

Personnel (September 1981):

Faculty:

S. Grimes

R. Lane

Post-doctoral:

H. Knox

G. Randers-Pehrson

\section{Graduate Student:}
S. Graham
P. Koehler
D. Resler 
Institution: Ohio University

Principal Investigator(s): D. Onley, L. Wright

Contract NO.: $\quad$ DE-ASO2-81ER10397

Title: "Theory of Electron Induced Reactions"

Address: Department of Physics ,

Ohio University

Clippingers Research Laboratories

Athens, $\mathrm{OH} \quad 45701$

Phone Number: (614)-594-.5740

Personnel (September 1981):

Facuity:

D. Onley

I. Wright

Post-doctoral:

Graduate Student:

P. Durgapal

F. Zamani-Noor 
Institution: University of Oklahoma

Principal Investigator(s): R. Petry

Contract No.: DE-AS05-80ER10495

Title: "Transitional Nuclei in the A 100 Region"

Address: Department of Physics and Astronomy University of Oklahoma

Norman, Ok lahoma 73019

Fhonc Number: (405) 325=3962

Personne1 (September 1981):

Faculty: R. Petry

Post-doctoral:

Graduate Student: H. Dejbakhsh 
Institution: Oregon State University

Principal Investigator(s): w. Loveland

Contract NO.: DE-ATO6-81ER70035

Title:- "Radioghemical Studies of Iow Energy and Relativistic Heavy Ion Reactions"

Address: Radiation Center Oregan State University Corvallis, OR 97331

Phone Number: (503)-754-2341

Personnel (September 1981):

Faculty:

W. Loveland

Post-doctoral:

Graduate Student:

I. Ghannam

R. Kraus 
92

Institution: Oregon State University

Principal Investigator (s): v. Madsen

Contract NO.: DE-AT06-81ERI0405

Title: "Theoretical Studies of Multistep Processes and Isospin Effects in Nuclear Scattering"

Address: Department of Physics

Oregon state Univoreity

Corvallis, OR. 97331

Phone Number: (5U3)-754-46s1

Personnel (September 1981):

Faculty:

v. Madsen

Post-doctoral:

Graduate Student: 
Laboratory: Pacific Northwest Laboratories

Division: Nuclear Physics

Personnel (September 1981):

Permanent Staff:

N. Ballou

H. Palmer

P. Reeder

R. Warner

Address: Battelle Memorial Institute Pacific Northwest Laboratory

P. 0. Box 999

Richland, WA 99352

Phone: (509) 375-2426 
Institution: University of Pennsylvania

Principal Investigator(s): F. Ajzenberg-Selove

Contract NO.: DE-ASO2-81ER02785

Title: "Compilation of Information on the Energy Levels of the Light Nuclei"

Address: Department of Physics

University of Pennsylvania

Philadelphia, PÁ 19104

Pliurie Number: $(215)-243-1293$

Personnel (September 1981):

Faculty:

F. Ajzenberg-Selove

Post-doctoral:

Graduate Student: 
Institution: University of Pennsylvania

Principar Investigator $(s):$ S. Frankel

Contract NO.: DE-ACO2-81ER04660

Title: . "Research in Very High Energy Nuclear Physics"

Address: $\quad$ Department of Physics University of Pennsylvania Philadelphia, PA 19104

Phone Number: (215)-243-3414

Personnel (September 1981):

Faculty:

S. Frankel

W. Frati

H. Brody

W. Molton

Post-doctoral:

\section{Graduate Student:}

P. Lindblom

M. Wood 
Institution: Princeton University

Principal Investigator $(s): R$. Naumann

Contract NO.: DE-ACO2-81ER02184

Title: "Nuclear Chemistry Project"

Address: Department of Chemistry Princeton University Princeton, NJ 00544

Phone Number: (FTS) $345-4372$

Personnel (September 1981):

Facuity:

$R$. Naumann

Post-doctoral:

Graduate Student:

r. Altzitzoglou 
Institution: Purdue University

Principal Investigator(s): P. Daly.

Contract No.: DE-AC02-81ER01672

Title: $\quad$ "Studies of Yrast and Continium States in A $=140-160$ Nuclei"

Address: Department of Chemistry ।

Purdue University

Chemistry Building

West Lafayette, IN 47907

Phone Number: (317)- 494-5328

Personnel (September 1981):

Faculty:

P. Daly

Z. Grabowski

Post-doctoral:

H. Helppi

M. Kortelahti

\section{Graduate Student:}

Y. Chung

J. McNeil1

J. Wilson 
Institution: Purdue University

Principal Investigator(s): N. Porile

Contract NO.: $\quad$ DE-ACO2-79ERO1505

Title: "Deexcitation Processes in Nuclear Reactions"

Address: Department of Chemistry

Purduge University

Chemistry Building

West Lafayette, IN 47907

Phone Number: (317)-494-2889

Personnel (September 1981):

Faculty:

N. Porile

Post-doctoral:

S. Agarwal

D. Bujak

Graduate Student:

J. Herrmann

D. Klingensmith

Y. Onkubo

c. Wang 
Institution: Purdue University

Principal Investigator $(s)$ : R. M. Steffen

Contract No.: $\quad$ DE-ACO2-81ER02875

Title: "Study of Muonic Atoms"

Address: Department of Physics

Purdue University

West Lafayette, IN 47907

Phone Number: (317)-494-8112

Personne 7. (September 1981):

Faculty:

R. M. Steffen

Post-doctoral:

Y. Tanaka

Graduate Student: 
Institution: Rensselaer Polytechnic Institute

Principal Investigator(s): D. Sperber

Contract NO.: DE-ACO2-81ER3463

Title: "Theoretical Studies of Heavy Ion Collisions at Low and Intermediate Energies Using Macroscopic Models"

Address: Department of Physics

Rensselaer Polytechnic Instilule Troy, NY 12181

Phone Number: (518)-270-6418

Personnel (September 1981):

Faculty:

D. Sperber

Post-doctoral:

B. Samanta

M. Zielinka-Pfabe

\section{Graduate Student:}




\section{Institution: Rice University}

Principal Investigator(s): G. Phillips

Contract No.: DE-AS05-81ER40032

Title: $\quad$ Nuclear Structure at Intermediate Energies"

Address: $\quad$ T. W. Bonner Nuclear Laboratories Rice University

Houston, TX 77001

Phone Number: (713)-528-2159

Personnel (September 1981):

Faculty:

S. Baker

I. Duck

G. Mutchler

G. Phillips

\section{Post-doctoral:}

J. Buchanan

J. Clement

H. Viereck

J. Windish

\section{Graduate Student:}
W. Madigan
C. Naudet
G. Pepins
S. Turpin
E. Uthland 
Institution: University of Rochester

Principal Investigator(s): J. French

Contract No.: DE-AC02-81ERO217I

Title: "Nuclear Structure Theory"

Address: Department of Physies and Astronomy University of Rochester Rochester, NY 14627

Phone Number. $(715)=275-4359$

Personnel (September 1981):

Faculty:

J. French

D. Koltun

Post-doctoral:

V. Kota

A. Saharia

Graduate Student:

S. $R a b$ 
Institution: University of Rochester

Principal Investigator(s):- J. Huizenga

Contract NO.: $\quad$ DE-ACO2-81ER03496

Title: Studies of Heavy Ion Reactions and Transuranic Nuclei

Addres5: Nuclear Structure Research Laboratory University of Rochester

Rochester, NY 14627

Phone Number: (716)-275-4941

FTS $430-4941$

Personnel (September 1981):

Faculty:

J. Birkelund

J. Huizenga

W. Schrọder

Post-doctoral:

W. Wilcke

H. Wollersheim

\section{Graduate Student:}
M. Butler
A. Hoover
J. Kosky
L. Tubbs 
Laboratory: Rockwell International

Division: Nuclear Physics

Personnel (September 1981):

Permanent Staff:
H. Farrar
D. Kneff

B. Oliver
Address: Rockwell International Corporation Energy Sys tems Group

P. 0. Box 309

Canoga Park, CA 91304

Phone: (213) 341-1000 
Institution: University of South Carolina

Principal Investigator(s): F. Avignone

Contract NO.: DE-AS09-81ER10434

Title: "High Resolution Positron $Q$-value Measurements and Nuclear Structure Studies far from the Stability Line"

Address: Department of Physics and Astronomy University of South Carolina Columbia, SC 29208

Phone Number: (803)-777-4121

Personnel (September 1981):

Faculty:

F. Avignone

Post-doctoral:

H. Noma

Graduate Student: 
Institution: Stanford University

Principal Investigator(s): R. Hofstadter, E. Hughes

Contract NO.: DE-AT03-80ERI0688

Title: "Equipment for Study of Rare Muon Decays at LAMPF"
Address: w. W. Hansen Laboratories of Physics Stanford University 3 tanford, CA 94305

Phone Number: (415)-479-0281

Personnel (September 1981):

Faculty:

R. Hofstadter

E. Hughes

Post-doctorai:

Graduate Student:

s. Wilson 
Institution: Temple University

Principal Investigator(s): w. McFarlane

Contract NO.: DE-ACO2-81ER0 3539

Title: "Experimental Investigations in Particle Physics at Intermediate Energies"

Address: Department of Physics

Temple University

Philadelphia, PA 19122

Phonen Number: $\quad(215)-787-7730$

Personnel (September 1981):

Facuity:

L. Auerbach

V. Highland

w. McFarlane

Post-doctoral:

Graduate Student:

F. Gaille

E. Jastrzembski 
Institution: Tennessee Technological University

Principal Investigator(s): R. Kozub

Contract NO.: DE-ASO5-79ER10335

Title: "Structure of Neutron Rich Nuclei Using Inverse Heavy Ion Reactions"

Address: Department of Physics

Tennessee Technological University

Cookeville, TN 38501

Phone Number: (615)-528-3479

Personnel (September 1981):

Faculty:

R. Kozub

Post-doctoral:

Graduate Student: 
Institution: Tennessee Technółogical University

Principal Investigator(s): J. Mateja

Contract NO.: DE-AS05-80ER10714

Title: "The Dependence of Heavy Ion Resonance Formation on the Nuclear Structure of the Entrance Channel Nuclei"

Address: Department of Physics Tennessee Technological University Cookeville, TN 38501

Phone Number: (615)-528-3483

Personnel (September 1981):

Faculty:

J. Mateja

Post-doctoral:

Graduate Student: 
Institution: University of Tennessee

Principal Investigator(s): C. Bingham, L. Riedingex

Contract NO.: DE-ASO5-79ER04936

Title: Nuclear spectroscopic studies:

Address: Department of Physics and Astronomy University of Tennessee

Knoxville, 'lenthessee 37916

Phone Number: (615) 974-3342

Personnel (September 1981):

Faculty:

C. Bingham

L. Riedinger

M. Guidry

Post-doctoral:

H. Owen

Graduate Student:

L. Courtney

P. Luk

T. Nicols 
Institution: Texas A\&M University

Principal Investigator $(s)$ : R. Bryan, B. VerWest

Contract NO.: DE-ASO5-81ER05223

Title: "Intermediate Energy Nuclear Theory"

Address: Department of Physics Texas A\&M University College Station, TX 77843

Phone Number: (713)-845-5111

\section{Personne1. (September 1981):}

Faculty:

R. Bryan

R. Clark

C. Dominguez

H. Garcilazo

B. Verwest

Post-doctoral:

L. Litsch

Graduate Student:

J. McClain 
Institution: Texas A\&M University

Principal Investigator(s): $\mathrm{L}$. Northcliffe

Contract NO.: DE-ASO5-76ER0 4449 Title: "Study of the Neutron-Proton Interaction in the 300-700 MeV Energy
Region"

Address: Cyclotron Institute Texas A\&M University

College Station, TX 77843

Phrnne Number: $\quad(71.3)-845-1411$

Personnel (September 1981):

Faculty:

J. Hiebert

R. Kenefick

I. Northcliffe

Post-doctoral:

T. Bhatia

G. Glass

\section{Graduate Student:}

W. Tippens 
Institution: Texas A\&M University

Principal Investigator(s): H. Schuessler . .

Contract NO.: . DE-AṢO5-80ERI0578

Title: "Hyperfine Structure and Isotope Shift Measurements of Short-Lived Elements by Laser Spectroscopy"

Address: Department of Physics Texas A\&M University College Station, TX 77843

Phone Number: (713)-845-5455

Personnel (September 1981):

Faculty:

H. Schuessler

Post-doctoral:

J. Parker

Graduate Student:

w. Pruitt 
Institution: Texas A\&M University

Principal Investigator(s): D. Youngblood

Contract NO.: DE-AS05-80ERI0565

Title: "Cyclotron-Based Nuclear Science"

Address: Cyclotron Institute Texas A\&M University

College Station, $\mathrm{TX} \quad 77843$

Phone Number: (713)-845-1411

Personnel (September 1981):

Faculty:

J. Bronson

R. Schmitt

D. Haenni

R. Tribble

T. Izumoto

B. Laune'

D. Youngblood

D. May

K. Nagatani

N. Namboodiri

J. Natowitz

L. Northoliffo

Post-doctoral:
R. Choudhury
N. Takahashi
U. Garg
E. Ungricht
D. Gonthier
Y. Lui
G. Mouchaty
S. Nath
R. Neese
J. Sullivan

Graduate Student:
L. Adler
J. Doumas
K. Hagel
M. Parkin
H. Simon
D. Tanner
H. Woolverton 
Institution: University of Texas

Principal Investigator(s): G. Hoffmann

Contract NO.: DE-AS05-81ERI0638

Title: "Nuclear Structure Studies using the High" Resolution Spectrometer at LAMPF"

Address: Department of Physics

University of Texas

Austin, TX 78712

Phone Number: (515)-417-1353

Personnel (September 1981):

Faculty:

G. Hoffmann

L. Ray

Post-doctoral:

M. Barlett

Graduate Student:

R. Fergerson

J. Marshall

E. Milner 
Institution: University of Texas

Principal Investigator(s): B. Hoistad

Contract NO.: DE-AS05-80ER10706

Title: "Experimental Research in Nuclear Physics at Intermediate Energies"

Address: Department of Physics University of Texas

Austin, TX 78712

Phone Number: (512)-471-1064

Personnel (September 1981):

Faculty:

B. Hoistad

1

Pusl-doctoral:

Graduate Student: 
Institution: University of Texas

Principal Investigator(s): c. Moore

Contract NO.: DE-ASO5-81ER05224

Title: "Research in Experimental Nuclear Physics"

Address: Department of Physics

University of Texas

Austin, TX 78712

Phone Number: (512)-471-1236 (FTS-734-5974)

Personnel (September 1981):

Faculty:

c. Moore

Post-doctora?:

C. Goulding

\section{Graduate Student:}

K. Boyer

W. Cottingame

S. Elstan

C. Harvey

R. Kaziah

B. Nelson 
Institution: University of Texas at Austin

Principal Investigator (s): P. Riley

Contract No.: DE-AC05-81ER40030

Title: "Medium Energy Measurements of N-N Parameters"

$\begin{array}{ll}\text { Address: } & \text { Department of Physics } \\ & \text { University of Texas } \\ & \text { Austin, TX } 78712\end{array}$

Phone Number: (512)-471-5957

Personnel (September 1981):

Faculty:

P. Riley

Post-doctoral:

C. Hollas

\section{Graduate Student:}

D. Cremans

R. Rodebaugh 
Institution: University of Texas at Austin

Principal Investigator(s): T. Tamura

Contract NO.: DE-ACO5-8IER4003I

Title: "Research in Theoretical Nuclear Physics"

Addres5: Department of Physics

University of Texas

Austin, TX 78712

Phone Number: (512)-471-1353

Personnel (September 1981):

Faculty:

w. Coker

T. Tamura

T. Udagawa

Post-doctoral:

$\because . Y$. Kondo

Y. Tzeng

\section{Graduate Student:}

I. Lee

v. Pedroçchi 
Institution: Vanderbilt University

Principal Investigator(s): J. Hamilton

Contract NO.: DE-ASO5-79ERO5034

Title: "Experimental Nuclear Structure Research via In-Beam Spectroscopy, Transfer Reactions, Heavy-Ion Collisions"

Address: Department of Physics and Astronomy

Vanderbilt University

Nashville, TN 37235

Phunte Number: (615)-32.2-2828

Personnel (September 1981):

Faculty:

R. Albridge

J. Hamilton

C. Maguire

R. Piercey

A. Ramayya

Post-doctoral:

L. Cleemann

S. Panchol

R. Soundranayagam

H. Yamada

\section{Graduate Student:}

M. Barclay

M. Herath-Banda

w. Ma 
Institution: Vassar College

Principal Investigator(s): R. Stearns

Contract NO.: DE-ACO2-77ERO4195

Title: "Measurement of Lambda and Sigma Hypernuclear States in Nuclei"

Addfess: Department of Physics \& Astronomy Vassar College Poughkeepsie, NY 12601

Phone Number: (914)-452-7000

Personnel (September 1981):

Faculty:

R. Stearns

Post-doctoral:

Graduate Student: 
Institution: Virginia Polytechnic Institute and State University

Principal Investigator(s): R. Arndt, L. Roper

Contract No.: DE-AS05-81ER04928

Title: "Partial Wave Analyses of Scattering Reactions: Below 2 GeV"

Address: $\quad$ Department of Physics

Virginia Polytechnic Institute and State University

Blacksburg, VA 24061

Phone Number: (703)-961-5353

Personnel (September 1981):

Faculty:

R. Arndt

L. Roper

Post-doctoral:

M. Manley

Graduate Student:

K. Hashimoto 
Institution: University of Virginia

Principal Investigator(s): . J. McCarthy

Contract NO.: DE-ASO5-80ERI0633

Title: $\quad$ Electron and Pion Interactions with Nuclei

Address: $\quad$ Department of Physics University of Virginia McCormick Road Charlottesville, VA 22901

Phone Number: (804)-924-3781

Personnel (September 1981):

Faculty:

J. Källne

J. McCarthy

B. Norum

R. Whitney

Post-doctoral:

R. Altemus

D. Day

L. Orphanos

Graduate Student:

G. Mechtel

J. Miller

w. Savansanbon •

T. Veng

J. Wise

N. Wright 
Institution: University of Virginia

Principal Investịgator $(s):$ R. Minehart, K. Ziock

Contract No.: DE-AS05-81ER0 4043

Title: "Experiments on the Nuclear Interactions of Pions"

Address: $\quad$ Department of Physics University of Virginia

Charlottesville, VA 22903

Phone Number: (804)-924-3781.

Personnel (September 1981):

Faculty:

R. Minehart

K. Ziock

Post-joctoral:

J. Boswell

W. Stephens

Graduate Student:

R. Marshall 
Institution: University of Washington, Seattle

Principal Investigator(s): E. Henley

Contract No.: DE-AC06-76ER01388

Title: Theoretical Nuclear and particle Physics

Address: $\quad$ Institute for Nuclear Theory University of Washington

Seattle, WA 98195

Phone Number: (206)-543-2770

Personnel (September 1981):

Faculty:

E. Henley

G. Miller

R. Puff

L. Wilets

Post-doctoral:

R. Goldflam

P. Hoodbhoy

W. Hwang

\section{Graduate Student:}

I. Carson

M. Morgan 
Institution: University of Washington, Seattle

Principal Investigator $(s):$. Vandenbosch

Contract NO.: DE-AC06-79ER01388

Title: $\quad$ University of Washington Nuclear Physics Laboratory Research and Operations",

Address: Nuclear Physics Laboratory

University of Washington

seattle, WA 98195

Phone Numher: (206)-543-4080

Personnel (September 1981):

Faculty:

E. Adelberger

J. Blair

D. Bodansky

J. Cramer

G. Furwe11

I. Halpern

A. Lazzarini

F. 3clumidl

K. Snover

Post-doctoral:

T. Trainor

R. Vandenbosch

W. Weitkamp

M. Doss

D. Dowe11

M. Hindi

Graduate Student:
T. Chupp
S. Kellogg
K. Davits
M. Khandaker
G. Feldman
K. Lesko
S. C.i11
D. Tingk.
D. Holmgren
R. Loveman
C. Hoyle
A. Ray
Z. Iqbal
R. Von Lintig 
Institution: Washington University, st. Louis

Principal Investigator(s): D. Sarantites

Contract NO.: DE-ACO2-81ER0 4052

Title: "Investigations of Nuclear structure and Nuclear Reactions Induced by Complex Projectiles"

Address: Department of Chemistry

Washington University

St. Louis, MO 63130

Phone Number: (314)-889-6504

Personnel (September 1981):

Faculty:

D. Sarantites

Post-doctoral:

F. Dilmanian

J. Jääskeläinen

\section{Graduate Student:}

R. Woodward 
Institution: University of Wisconsin, Madison

Principal Investiḡator(s): L. Anderson

Contract NO.: DE-ACO2-81ER40001

Title: "Research and Development on Optically Pumped Polarized Ion Sources"

Address: Department of Physics University of Wisconsin 1150 University Avenue

Madison, WI 53706

Phone Nunter: (608)-262-3077

Personnel (September 1981):

Faculty:

L. Anderson

Post-doctoral:

Graduate Student:

J. Cusma

D. Swanson 
Institution: University of Wisconsin - Madison

Priricipal Investigator(s): L. Knutson

Contract NO.: DE-ACO2-81ERIOU10

Title: "Search for Charge Symmetry Violation in n-p Scattering"

Addres5: Department of Physics University of Wisconsin 1150 University Avenue Madison, WI 53706

Phone Number: (608)-262-3096

Personnel (September 1981):

Faculty:

L. Knutson

P. Quin

Post-doctoral:

Graduate Student:

G. Orr

J. Sowinski 
Institution: University of Wisconsin, Madison

Principal Investigator(s): H. Richards

Contract No.: $\quad$ DE-ACO2-81ER40010

Title: "Research in Nuclear Physics"

Address: Department of Physics University of Wisconsin 1150 University Avenue Madicon, WI 53760

Phone Number: $(608)-262-3822$

Personnel (September 1981):

Faculty:

w. Haeberli

L. Knutson

P. Quin

Y. Rao

H. Richards

Z. Wang

J. The

Post-doctoral:

J. Billen

D. Mavis

S. Sen

Graduate Student:
M. Barker
S. Riedhauser
R. Bigelow
J. Soderstrum
G. Caskey
P. Colby
C. Gassett
J. Kammervad
G. Orr 
Institution: University of Wyoming

Principal Investigator(s): R. Kunselman

Contract NO.: DE-ACO2-81ER02197

Title: "Mesonic Atoms"

Address: Department of Physics and Astronomy University of Wyoming

P. O. Box 3905, University Station

Laramie, WY 82071

Phone Number: (307)-766-4366

Personnel (September 1981):

Faculty:

R. Kunselman

Post-doctoral:

Graduate Student:

M. Manley 
Institution: Yale University

Principal Investigator(s): D. Bromley

Contract NO.: $\quad$ DE-ACO2-76ERO3074

Title:

"Nuclear Physics Research Operations and Development at the Wright Nuclear Structure Laboratory"

Addres :

A. W. Wright Nuclear Structure Laboratory

Yale University

272 Whitney Avenue

New Haven, CT 06520

Phone Number: (202)-436-3026

Personnel (September 1981):

Faculty:

C. Bockelman

S. Sanders

M. Strayer

D. Bromley

R. Weller

J. Cizewski

F. Firk

J. Greenberg

F. Iachello

Post-doctorat:
A. Ahmed
M. Anthony
E. Fehr
N. Tsoupas
M. Weller
M. Gai
W. Grèiner
A. Howard
R. Keddy
P. LaMarche
R. NaLis
s. Willett

Graduate Student:
B. Balantekin
A. Hayes
E. Schloemer
S. Catto
A. Champagne
J. Kruk
R. Shivakumar
M. Cunningham
S. Kuyucak
J. Schweppe
S. Lee
S. Sterbeny
B. Dichter
J. Manoyan
J. Thomas
M. Drigert
J. O'Conner
S. Umar
M. Greene
M. Rosenthal
T. Wang
M. Ruscev
T. Zouros
R. Hatch 
Institution: Yale university

Principal Investigator(s): $\quad$ v. Hughes

Contract NO.: DE-ACO2-81ER03075

Title: "Medium Energy Elementary Particle Physics"

Address: Department of Physics

Yale University

New Haven, CT 06520

Phone Number: (203)-436-3566

Personnel (September 1981):

Faculty:

P. Egan

V. Hughes

D. Lu

P. Souder

Post-doctoral:

T. Gay

F. Mariam

R. Schaefer

Graduate Student:
P. Bolton
G. Cates
C. Gardner
M. Green 


\section{United Statos}

Department of Energy

Washington, DC 20545

Postage and Fees Paid

U.S. Department of Energy

DOE-350

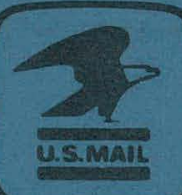

\section{ficial Busines}

nalty for Private Use, $\$ 300$

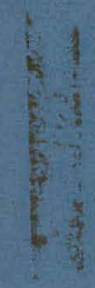

